\title{
Recovery from Alopecia Universalis after Bilateral Lung Transplantation
}

\author{
Imran Nizami ${ }^{1}$, Tufail Khizer ${ }^{1}$, Fayyaz Baig ${ }^{1}$ and Rozeena Huma ${ }^{2 *}$ \\ ${ }^{1}$ Organ Transplant Center, King Faisal Specialist Hospital and Research Center, Saudi Arabia \\ ${ }^{2}$ Department of Clinical Research, King Faisal Specialist Hospital and Research Center, Saudi Arabia
}

Submission: January 18, 2022; Published: January 27, 2022

*Corresponding author: Rozeena Huma, Research consultant, Clinical Research Department, King Faisal Specialist Hospital and Research Center, MBC: 117, 11211, Riyadh, Saudi Arabia

\section{Abstract}

Alopecia areata (AA) is an immune-mediated form of hair loss that occurs in all ethnic groups, ages, and both sexes, with an estimated lifetime risk of $1.7 \%$ among the general population. Approximately $15-25 \%$ of all AA cases progress to Alopecia universalis (AU), which is a complete loss of scalp and body hair. Complete recovery from $\mathrm{AU}$ is rare $(<10 \%)$. We report a case of AU in a patient who was referred to us for lung transplantation due to advanced bronchiectasis. The patient recovered from AU within three months of bilateral lung transplant using standard triple immunosuppressive therapy. To our knowledge, this is the first case report of AU recovery with standard immunosuppression use after lung transplantation.

Keywords: Alopecia universalis; Alopecia totalis; Alopecia areata; Lung transplantation

\section{Introduction}

Alopecia Areata (AA) is a chronic inflammatory disease that affects the hair follicle and sometimes the nail. Alopecia universalis $(\mathrm{AU})$ is an immune-mediated form of hair loss. Onset can be at any age and there is no known race or sex preponderance $[1,2]$. Alopecia Areata usually presents as patches of hair loss on the scalp, although any hair-bearing skin can be involved. Hair follicles are preserved in alopecia areata and the potential for recovery of hair growth is maintained, even in longstanding disease. Spontaneous remission within one year occurs in 34-50\% of the cases. Almost all affected patients will experience more than one episode of disease recurrence, and $15-25 \%$ will progress to either a total loss of scalp hair (Alopecia Totalis, AT) or a total loss of entire scalp and body hair (Alopecia universalis, AU), from which full recovery is unusual $(<10 \%)[2,3]$.

No treatment has been shown to have a significant long-term benefit compared to placebo.

A number of treatments can induce hair growth in AA but none has been shown to alter the long-term course of the disease. These treatments include but are not limited to topical, intralesional and systemic steroids, contact immunotherapy, phototherapy, minoxidil, dithrinol, and cyclosporine [4]. Contact immunotherapy is the best-documented treatment for severe alopecia areata but it is not widely available, involves multiple visits to the hospital over several months and stimulates cosmetically worthwhile hair regrowth in $<50 \%$ of patients. It is the only effective treatment in $\mathrm{AT} / \mathrm{AU}$, although the response rate is low [4].

\section{Case Report}

A 45-years-old Saudi male was seen for the first time at KFSH $\&$ RC in May 2014. He was referred to our unit for lung transplant secondary to advanced bronchiectasis. Patient had progressive dyspnoea on exertion for the last 10 years. At the time of referral, he was oxygen dependent with dyspnoea on performing activities of daily life. He developed Alopecia Areata (AA) at the age of 25 years. He consulted a local dermatologist who prescribed topical steroid injections with some improvement. However, he stopped the treatment because of intolerable pain of the injections. He experienced gradual progression of AA to Alopecia universalis (AU) over a period of 2-3 years (Figure 1). He had past medical history of non-autoimmune hypothyroidism and azoospermia. For Hypothyroidism he received thyroid hormone replacement therapy.

On physical and systemic examination revealed normal appearance of nails, absence of scalp, facial and body hair, crackles in bilateral lung fields. The bronchiectasis workup was negative 
for cystic fibrosis, immune deficiency and immotile cilia syndrome. He underwent standard work up to evaluate his candidacy for lung transplantation. Based on his advanced bronchiectasis that had not responded to multiple medical therapies, he was accepted as a candidate for bilateral lung transplantation and placed on the lung transplant waiting list.

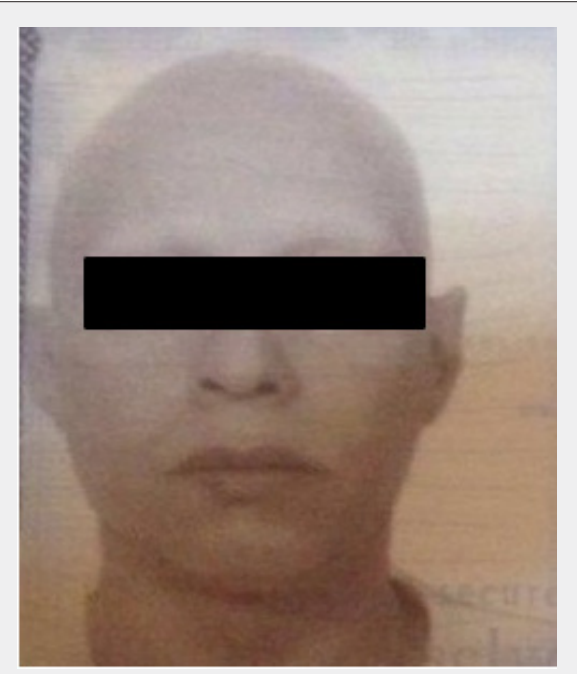

Figure 1: Photo of patient displaying an absence of facial and scalp hair.

The patient underwent bilateral lung transplantation on 31 August 2014. The immunosuppression included Prednisone, Mycophenolate mofetil, and Tacrolimus. In addition, he received antimicrobial prophylaxis comprising Sulfamethoxazole/ Trimethoprim three times per week, Val ganciclovir daily, and Itraconazole daily. Itraconazole was discontinued after three months and Val ganciclovir was stopped at 12 months after surgery. He was maintained on triple immunosuppression and Sulfamethoxazole/Trimethoprim. His thyroid hormone replacement remained unchanged.

His surveillance biopsy showed two episodes of A2 rejection 1 and 3 months post lung transplant for which he received IV Methyl prednisone $7 \mathrm{mg} / \mathrm{kg}$ for 3 days as out-patient. Subsequent biopsies did not show any evidence of acute cellular rejection.

One-month post treatment for his bilateral lung transplant, he noticed regrowth of scalp and body hair. A near complete recovery of scalp hair within 3 months was observed after bilateral lung transplant. Dose of Prednisone was reduced to $5 \mathrm{mg}$ daily, he noticed partial loss of scalp hair upon this change of dose. No further hair loss was noted. He remained stable for 2 years post bilateral lung transplant with stable lung allograft function and stable scalp and body hair distribution (Figure 2).

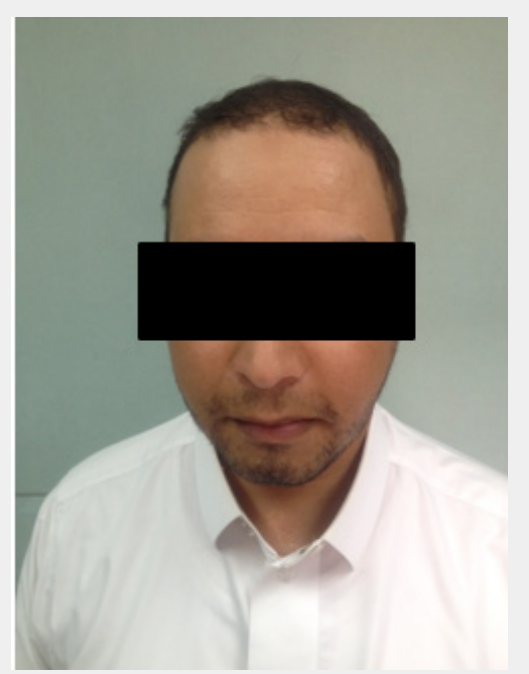

Figure 2: Photo of patient showing regrowth of facial and scalp hair. 


\section{Discussion}

In this case report, we describe a patient with $\mathrm{AU}$ who experienced recovery of scalp and body hair after bilateral lung transplantation. The patient was given standard triple immunosuppression therapy as per our post lung transplantation protocol, including oral Prednisone in a tapering dose, Tacrolimus, and Mycophenolate mofetil [5,6]. Our patient has maintained hair growth at the midterm follow up of two years' post-transplantation. At the end of two years, he is on maintenance dose of Prednisone 5mg daily, Mycophenolate 1000mg twice daily and Tacrolimus trough level is maintained between 6 and $8 \mathrm{ug} / \mathrm{L}$.

To our knowledge, this is the first case report of the recovery from $\mathrm{AU}$ after bilateral lung transplant with the use of triple immunosuppression. This case may open the avenue of further research in the management of this unpredictable disease. Combination therapy with reduced doses of individual medications may have better tolerance and reduced toxicity. The optimum dose of the individual medications yet to be identified.

\section{Conclusion}

This case report shows a patient fully recovered from Alopecia Totalis from combination of immunosuppressive therapy. This patient did not have any significant adverse effects from the immunosuppressive regimen. However, the appropriate minimum dose of immunosuppression required to reverse alopecia totalis remains to be determined.
Informed Consent was Obtained as per ethics.

\section{Conflict of Interest}

We, authors, would like to state that we do not have any commercial association for conducting or publicizing the study described in the manuscript that might pose or create a conflict of interest with the information presented in the manuscript.

\section{References}

1. McDonagh AJG, Tazi-Ahnini R (2002) Epidemiology and genetics of alopecia areata. Clin Exp Dermatol 27(5): 405-409.

2. Antonella Tosti, Sara Bellavista, Matilde Iorizzo (2006) Alopecia areata: A long term follow-up study of 191 patients. J Am Acad Dermatol 55(3): 438-441.

3. MacDonald Hull SP, Wood ML, Hutchinson PE, Sladden M, Messenger AG, et al. (2003) Guidelines for the management of alopecia areata. Br J Dermatol 149(4): 692-699.

4. Messenger AG, McKillop J, Farrant P, McDonagh AJ, Sladden M (2012) British Association of Dermatologists' guidelines for the management of alopecia areata. Br J Dermatol 166(5): 916-926.

5. Knoop C, Haverich A, Fische S (2004) Immunosuppressive therapy after human lung transplantation. Fische Eur Respir J 23(1): 159-171.

6. Scheffert JL, Raza K (2014) Immunosuppression in lung transplantation. J Thorac Dis 6(8): 1039-1053. 